\title{
A Novel Congestion Control Mechanism with Accelerating Effect
}

\author{
Vivek Singhal \\ College of Engineering Roorkee \\ Roorkee \\ Uttrakhand, India
}

\author{
A.K.Vatsa \\ School of Computer Engg. \& IT \\ Shobhit University \\ Meerut, Uttar Pradesh, India
}

\begin{abstract}
The tremendous growth of wireless network demands the need to meet different multimedia applications available over the wireless networks. Network congestion occurs when a link or a node is carrying an excessive amount of data because of which the quality or service deteriorates and these demands and allocations lead to optimized rate based control regulation through queuing theory. Earlier works on rate control protocols does not emphasize on different RCP wireless practical situations which may have varying or constant link and packet acceptance capacity, bandwidth coordination, acceleration maintenance etc. In this paper, we purpose a novel, methodology for rate based congestion control in wireless network. Thus, we proposed an algorithm of a range based capacity wireless network with different conditions for maintaining the acceleration and traffic in terms of capacity of processor nodes and link capacity
\end{abstract}

\section{General Terms}

Congestion Control, Wireless Networks.

\section{Keywords}

RCP (Rate Control Protocol), XCP (Explicit Control Protocol), Wireless network, Congestion.

\section{INTRODUCTION}

\subsection{Wireless Network}

Wireless network refers to any type of computer network that is implemented without the use of wires between intermediate nodes [6] and having features like fast deployment [16], cost reduction, vulnerability, easy sharing of data, fast communication, security and scalability. The applications of wireless networks include cellular telephony, wireless energy transfer, Wi-Fi, security systems and the problems involving the wireless network are high setup cost, limited range coverage, high susceptibility to interference, interception by unauthorized user, slow speed.

\subsection{Congestion Control}

Congestion occurs when the number of packets being transmitted through the network approaches the packet handling capacity of the network. Network congestion is caused by variety of factors, including network topology, bandwidth and usage patterns. Faulty hardware, network broadcasts and expansion also slow the networks. The measures for avoidance of network congestion require two major components i.e. a router mechanism to reorder or drop packets under overload and end-to-end flow control mechanisms designed into the end points which respond to congestion and behave appropriately.
Congestion control [15] involves the controlling traffic in a network to avoid oversubscription of any of the processing or link capabilities of the intermediate nodes and networks and taking resource reducing steps, such as reducing the rate of sending packets. The argument for flow completion time being the appropriate metric for congestion control is presented in [12].

\subsection{RCP}

The Rate Control protocol RCP[1] is a part of the 100x100 clean state project [17] and is an explicit feedback method in which a router maintains a constant rate $\mathrm{R}(\mathrm{t})$ for every flow on the network. Every router inserts $\mathrm{R}(\mathrm{t})$ and $\mathrm{RTT}$ in all packets moving on the link, then receiver sends back the smallest $R(t)$ and RTT back to the sender therefore less queue occupancy . In RCP [8], every router embeds same rate to all flows so as to do processor sharing. The RCP rate update equation (1) is given below:

$\mathrm{R} \mathrm{t}=\mathrm{R} \mathrm{t}-\mathrm{T}(1+\mathrm{T} / \mathrm{d} \quad \alpha \cdot \mathrm{CL}-\mathrm{yt} \quad-\beta \cdot \mathrm{qt} / \mathrm{d} / \mathrm{CL}$

where $\mathrm{d}$ is a moving average of the RTT measured across all packets (each RCP sender maintains its RTT estimate which it stamps in all outgoing data packets), $\mathrm{T}$ is the update interval(i.e., how often $\mathrm{R}(\mathrm{t})$ is updated) and is less than or equals $d, R(t-T)$ is the last rate, CL is the link-capacity, $y(t)$ is the measured aggregate input traffic rate during the last update interval, $q(\mathrm{t})$ is the instantaneous queue size, and $\alpha, \beta$ are parameters chosen for stability and performance.The problem lies here in RCP of either over-estimation or underestimation of the allocated rate $\mathrm{R}(\mathrm{t})$. Therefore the router divides the capacity into large number of flows hence link underutilization.

\section{$1.4 \mathrm{XCP}$}

$\mathrm{XCP}$ [9] is the explicit congestion control protocol which helps the routers to control flow rate hence high link utilization. XCP gives the routers a fine-grained control over sending rates of flows [13]. XCP enables the routers in the network to return explicit feedback back to the hosts and allows the routers in the network to continuously coordinate the sending speed of participating hosts. Every router routes the information related to increase or decrease of sending flow rate of the sender accordingly and receiver returns back the feedback. The XCP protocol adjusts the bandwidth on a per packet calculation. XCP routers do not separate between different data-flows, but make adjustments on each individual packet. The problem in the fact 
here is that XCP routers must have knowledge of the link capacities and internal queue statistics.

\subsection{Next Generation Wireless Applications}

New generation applications which are large in number, need high processing speed and large processors capacity to work upon when deployed in current Internet wireless scenario.So,need for the development and improvement of the techniques for reducing congestion in wired and wireless networks[10],[11] is of major concern and importance.Every processor node in the network has a limited capacity to accept the packets so rate of flow of packets to the processor nodes is to be managed likewise a particular router installed on the network can usually accomodate a fixed number of packets so different routers have a same varying packet accomodation capacity,this makes the management difficult,therefore different conditions analysis to make system more roboust is an important task.

\subsection{Problem Identification}

Wireless Network involved the problems of uneven utilization of processing or link capacities of the intermediate nodes on the network whereas RCP had the problems of link under utilization and acceleration maintenance and XCP clubs the difficulties in accumulating knowledge of link capacities and queue statistics in wireless networks. Therefore, we found the problems of bandwidth coordination and acceleration maintenance over varying or constant link capacity network and packet acceptance capacity of the node over wireless network.

\subsection{Paper Organization}

In this paper, we have organised it $\mathrm{n}$ chapters. Thus, In Chapter1 , we have discussed the Introduction. Based on literature review, topics like Rate Control Protocol(RCP),Coded Rate Control protocol(C-RCP), Proportional Integral Queue Independent RCP(PIQI-RCP), RCP with acceleration control(RCP-AC),RCP with small buffers, WRCP (Wireless Rate Control Protocol) we found some problems in these mechanism that we have discussed it in Chapter-2 entitled Related Work. In Chapter-3 we proposed the solution for problems under the heading of the proposed work. In Chapter-4 provides the conclusion and finally in Chapter-5 provides the future scope.

\section{RELATED WORK}

In [1], congestion control algorithm called Rate Control Protocol (RCP) - which emulates Processor sharing over a broad range of operating conditions. Here every router inserts R(t) and RTT in all packets moving on the link, then receiver sends back the smallest $\mathrm{R}(\mathrm{t})$ and RTT back to the sender therefore less queue occupancy. In this algorithm which is an explicit feedback method every router is assigned the same rate to all flows passing through it and also the calculations is not done on basis of packet but paper limitations involve about no implementation of RCP on wireless networks and over-estimation or underestimation of the allocated rate $\mathrm{R}(\mathrm{t})$.

In [3], the paper purpose a controller called Proportional Integral Queue Independent RCP (PIQI-RCP) which is heterogeneously stable, require small buffers at routers and does not move to the current fair bandwidth share in the network immediately. Proportional component helps in improving the system response time and restrict the queue size. Here new flows slowly increment rate of sending of feedback to the routers which are congested so, AFCT is lower than TCP and XCP but the problems found in this paper involved the difficulty in multi-link stability analysis of max-min congestion control and explicitfeedback deployment.

In [4], author extended RCP and proposed RCP-AC i.e. RCP with Acceleration controller which was stable over all operating conditions. Acceleration maintenance is a major issue in wireless networks so for this the we used equation (2) as in this paper which controls the maximum increase in aggregate traffic volume permitted in next time period. $\varphi=\alpha \mathrm{CLij}-\mathrm{y} \mathrm{t} \cdot \mathrm{d}+(\gamma \mathrm{B}-\beta \mathrm{q}(\mathrm{t}))-2$

where $B$ is the buffer size and $\gamma$ is the user-defined limit on acceleration, $\mathrm{CL}_{\mathrm{ij}}$ is the link capacity from a router $\mathrm{R}_{\mathrm{i}}$ to a particular node $\mathrm{P}_{\mathrm{ij}}, \alpha$ and $\beta$ are the stability and performance factors, $q(t)$ is the instantaneous queue size, $y(t)$ is the measured aggregate input traffic during the last update interval. The limitation of this RPC-AC is that it creates a trade off between how much acceleration the flows complete and buffer acceptance capacity and very complex.

Paper [5] purpose a Wireless Rate Control Protocol (WRCP), which uses an explicit feedback on precise capacity information over a collection tree. It uses a model that associates in terms of CSMA MAC for sensor networks. WRCP is a rate based protocol which achieves a max-min fair rate and tries to shorten the flow completion times by explicitly allocating rates based on available capacity in the network. This model is analogous to CSMA based wireless sensor network. It has fast flow completion times, good throughput and considerable packet delays. Limitation arises in this protocol with average capacity considered here not the actual capacity; more work on the wireless networks based on capacity is an open issue.

In Coded rate Control Protocol (C-RCP) [2] opinion a scheme for feedback in routers which works by limiting the transmission rate based on the packet erasure rate (PER), and makes use of this erasure coding to provide reliability for wireless links, using Luby Transform (LT) [14] and provides congestion control for lossy networks to increase the throughput of the network. Our aim is to get feedback only by this technique with slight modification that next insertion of output packet into a sliding window which slides whenever acknowledgement of a particular packet is received and output packet is generated with random selection of any number of packets not till half of packets.

In [7], a variant of RCP (rate control protocol) was developed that achieved $\alpha$ fairness when buffers are small, here it was shown that queue term is excluded from the RCP equation the stability increases, here in this paper a simple decentralized sufficient condition for local stability was established with aggregate flow through the resource, without knowledge of individual flow rates. The problem arises here is queue can't be excluded at all.

\section{PROPOSED WORK}

In this paper we purposed a Congestion Control mechanism through the bandwidth coordination and acceleration maintenance over varying or constant link capacity network and packet acceptance capacity of the node as in receiver capacity based network, the capacity of wireless network with RCP sender source server and receiver, and describe how it behave 
with different or same capacity based links and processor nodes and the solutions to it. In traditional wired networks, the notion of capacity is associated with a link existing between any two nodes. All flows traversing the link are assumed to linearly share the constant capacity of the link. In wireless networks, however, the capacity of a link is not constant, but rather is affected by activity on interfering links in its vicinity. Model for Congestion control mechanism is illustrated in Fig 1:

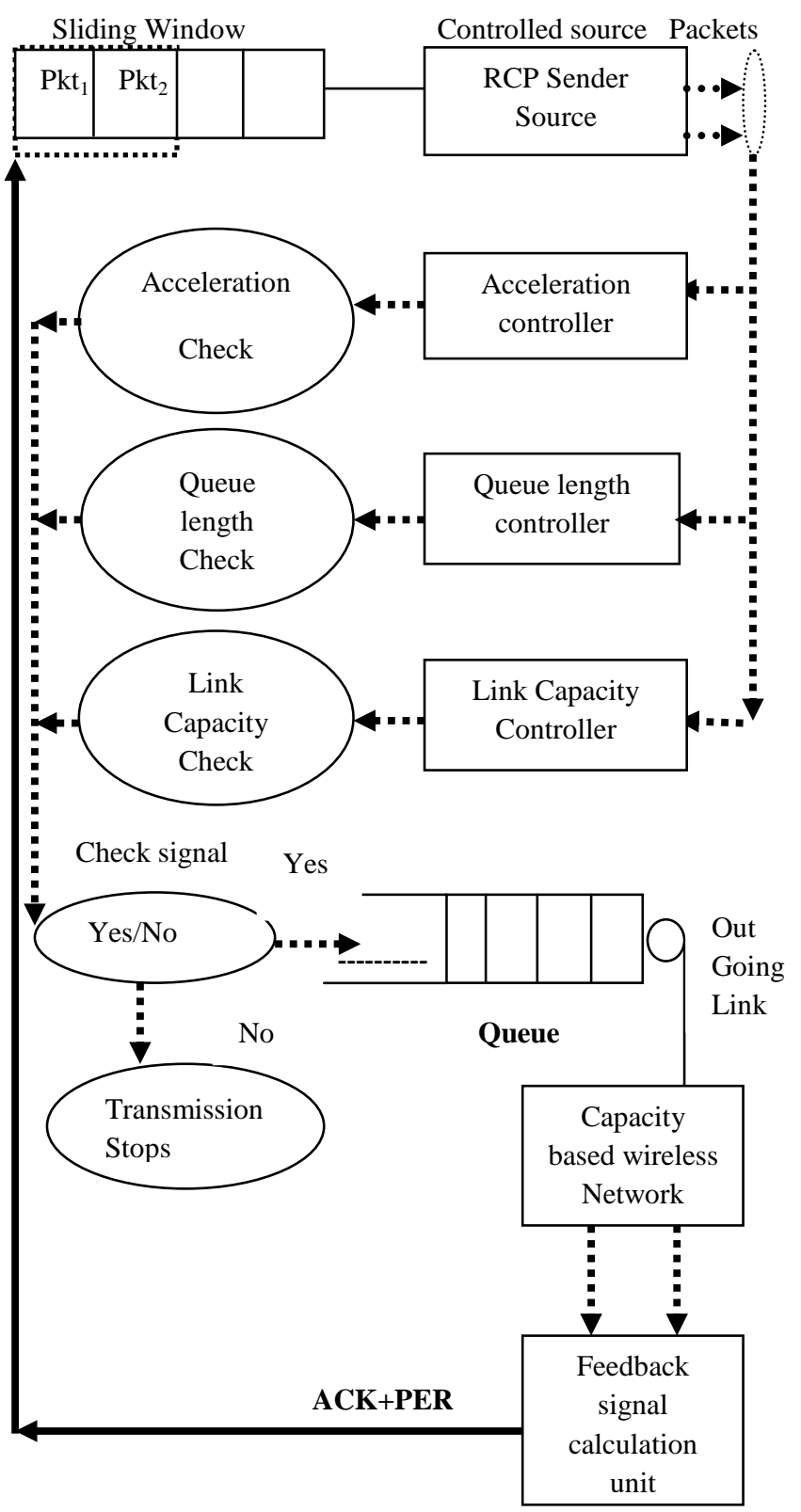

Fig 1: Model for Congestion Control Mechanism

Algorithm describes a range-capacity wireless network with RCP sender source and receiver. So, the common terms used in algorithms are $R_{i}(t)$ is the rate of flows by a router(i), $P_{i j}$ are the processor nodes having corresponding receiver capacities $C_{i j}, R_{i j}$ is the rate of flow to a particular processor node in $\mathrm{Mb} / \mathrm{s}, \mathrm{CL}_{\mathrm{ij}}$ is the link capacity from a router $R_{i}$ to a particular node $P_{i j}, q(t)$ is the instantaneous queue size at a router, $\mathrm{Rpkt}_{\mathrm{i}}, \mathrm{RTT}_{\mathrm{i}}, \mathrm{PER}_{\mathrm{i}}$ are the packet transmission rate, round trip time, PER estimate of packet ' $\mathrm{i}$ ', ACK[ij] is the acknowledgment of a particular processor node $\mathrm{P}_{\mathrm{ij}}$, where $\mathrm{ij}=$ number of recently sent packet and ranges from $1 \ldots . . . \mathrm{n}$, sliding window of size ' $\mathrm{W}$ ' and ' $\mathrm{N}$ ' is the number of packet to be transmitted from sliding window. The algorithm is described in phases as:

Phase I: Packet arrives from RCP sender source

* Sender start sending the packets controlled by RCP mechanism where $W$ is Maximum window size and $N$ is the number of packets $*$ /

a) Range-capacity network matrix of processor nodes $\mathrm{P}_{\mathrm{ij}}$ ' $\mathrm{s}$ in the range of a router ' $\mathrm{R}_{\mathrm{i}}$ ' (as it is capacity dependent network) is given by:

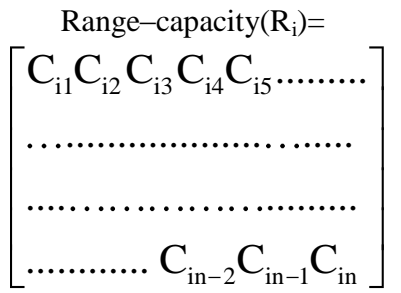

where $\mathrm{i}=1 \ldots \ldots \mathrm{n}$

selects the router ' $\mathrm{R}_{\mathrm{i}}$ ' and also, $\mathrm{P}_{\mathrm{ij}}$ 's in range of particular $\mathrm{R}_{\mathrm{i}}$ interfere each other

b) If $(\mathrm{N}==\mathrm{W})$ then Transmit the ' $\mathrm{N}$ ' packets on the network

c) If output packet (with $\mathrm{Rpkt}_{\mathrm{i}}, \mathrm{RTTpkt}_{\mathrm{i}}$ in packet header and data) arrives at router $R_{j}(t)$, then

$$
\mathrm{q}(\mathrm{t}) \leftarrow \text { output packet }
$$

d) $R_{i}$ maintains its flow of packets according to following cons-traints:-

$$
\begin{aligned}
& \text { If }\left(\left(\mathrm{Rpkt}_{\mathrm{i}}<\mathrm{R}_{\mathrm{i}}(\mathrm{t})\right) \& \&\left(\mathrm{RTTpkt}_{\mathrm{i}}<\mathrm{RTT}_{\mathrm{i}}\right)\right) \text { then } \\
& \mathrm{R}_{\mathrm{i}}(\mathrm{t}) \leftarrow \mathrm{Rpkt}_{\mathrm{i}} \\
& \mathrm{RTT} \leftarrow \mathrm{RTTpkt} \\
& \text { Calculate } \text { PER }_{\mathrm{i}} \\
& \}
\end{aligned}
$$$$
\text { Else If }\left(\left(\mathrm{Rpkt}_{\mathrm{i}}<\mathrm{R}_{\mathrm{i}}(\mathrm{t})\right) \& \&\left(\mathrm{RTTpkt}_{\mathrm{i}}>\mathrm{RTT}_{\mathrm{i}}\right)\right) \text { then }
$$$$
\{
$$$$
\mathrm{R}_{\mathrm{i}}(\mathrm{t}) \leftarrow \mathrm{Rpkt}_{\mathrm{i}}
$$$$
\text { Calculate } \mathrm{PER}_{\mathrm{i}}
$$$$
\}
$$

ElseIf $\left(\left(\mathrm{Rpkt}_{\mathrm{i}}>\mathrm{R}_{\mathrm{i}}(\mathrm{t})\right) \& \&\left(\mathrm{RTTpkt}_{\mathrm{i}}<\mathrm{RTT}_{\mathrm{i}}\right)\right)$ then

$\left\{\quad \mathrm{RTT}_{\mathrm{i}} \leftarrow \mathrm{RTTpkt}_{\mathrm{i}}\right.$

Calculate PER $_{\mathrm{i}}$

\}

Else 
do nothing

Phase II: Packet received by processor nodes $\mathbf{P}_{\mathrm{ij}}$

If $\left(\left(\mathrm{CL}_{\mathrm{ij}} \& \& \mathrm{C}_{\mathrm{ij}}\right)==\right.$ constant $)$ then

\{ all $\mathrm{P}_{\mathrm{ij}}$ 's in range of $\mathrm{R}_{\mathrm{i}}$ receive same number of packets

\}

Else If $\left(\mathrm{CL}_{\mathrm{ij}}=\neq\right.$ constant $\& \& \mathrm{C}_{\mathrm{ij}}==$ constant $)$ then

\{ If $\left(\mathrm{CL}_{\mathrm{ij}}==\right.$ large $)$ then

$\left\{\mathrm{R}_{\mathrm{ij}}(\mathrm{t}) \leftarrow\right.$ large

$\varphi \leftarrow$ small

\}

Else

$\left\{\mathrm{R}_{\mathrm{ij}}(\mathrm{t})\right.$ is small.

$\varphi \leftarrow$ large

\}

\}

Else If $\left(\mathrm{CL}_{\mathrm{ij}}==\right.$ constant $\& \& \mathrm{C}_{\mathrm{ij}}=\neq$ constant $)$ then $\left\{\right.$ If $\left(\mathrm{CL}_{\mathrm{ij}}==\right.$ large $)$ then

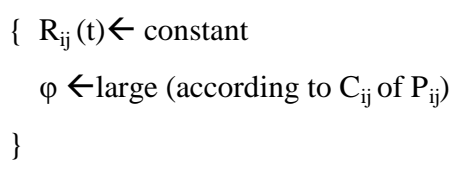

Else

\{

$\mathrm{R}_{\mathrm{ij}}(\mathrm{t}) \leftarrow$ constant

$\varphi \leftarrow$ small( according to $\mathrm{C}_{\mathrm{ij}}$ of $\mathrm{P}_{\mathrm{ij}}$ )

\}

$$
\}
$$

Else

\section{$\{\quad$ Buffer overflow \\ $\Phi \leftarrow$ very small}

\}

\section{Phase III: Acknowledgement from receiver nodes}

a) ACK [ij] with PER estimate send to the source.

b) Next output packet is selected based on PER estimate by :

$$
\begin{aligned}
& \mathrm{Pkt}_{31} \text { xor } \mathrm{Pkt}_{32} \text { xor } \mathrm{Pkt}_{33} \ldots \ldots \ldots \ldots \mathrm{Pkt}_{3 \mathrm{n}}=\mathrm{Pkt}_{3} \\
& \mathrm{Pkt}_{41} \text { xor } \mathrm{Pkt}_{42} \text { xor } \mathrm{Pkt}_{43} \ldots \ldots \ldots \ldots \mathrm{Pkt}_{4 \mathrm{n}}=\mathrm{Pkt}_{4}
\end{aligned}
$$

and inserts it into the sliding window

c) The window slides to next output packet.
The Algorithm phases are illustrated in Fig 2. As below:

Phase I:-

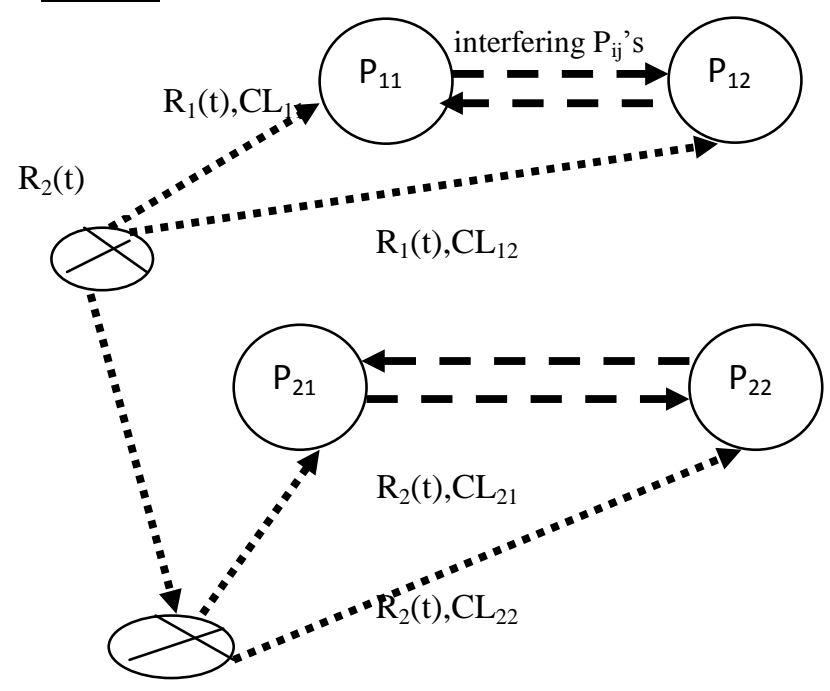

$\mathrm{R}_{2}(\mathrm{t})$

(a) Range capacity Network

RCP Sender Source

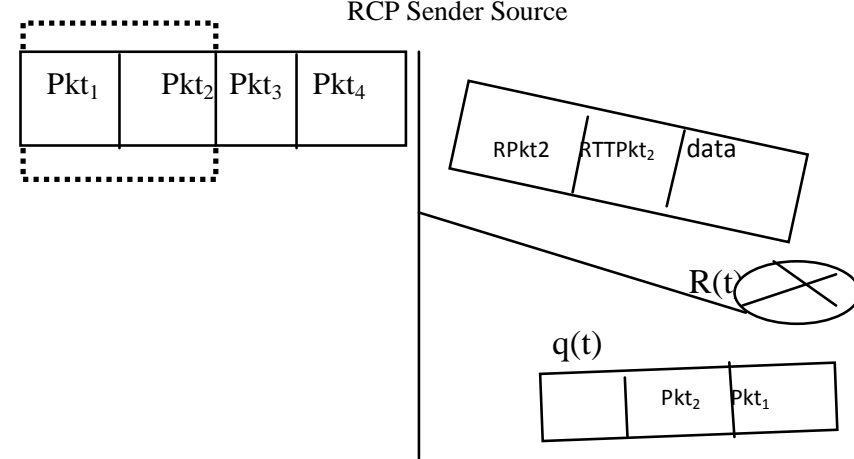

(b) \& (c)

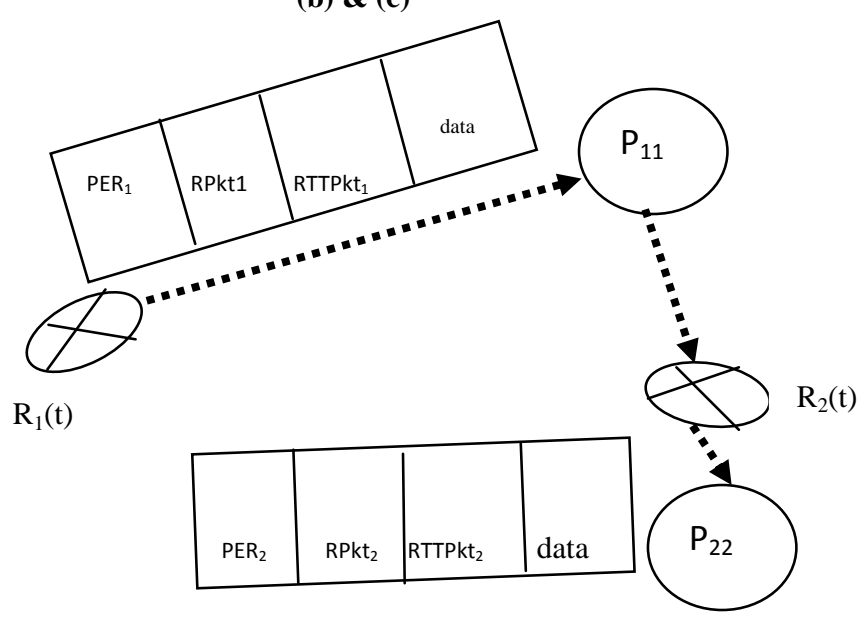

(d) 

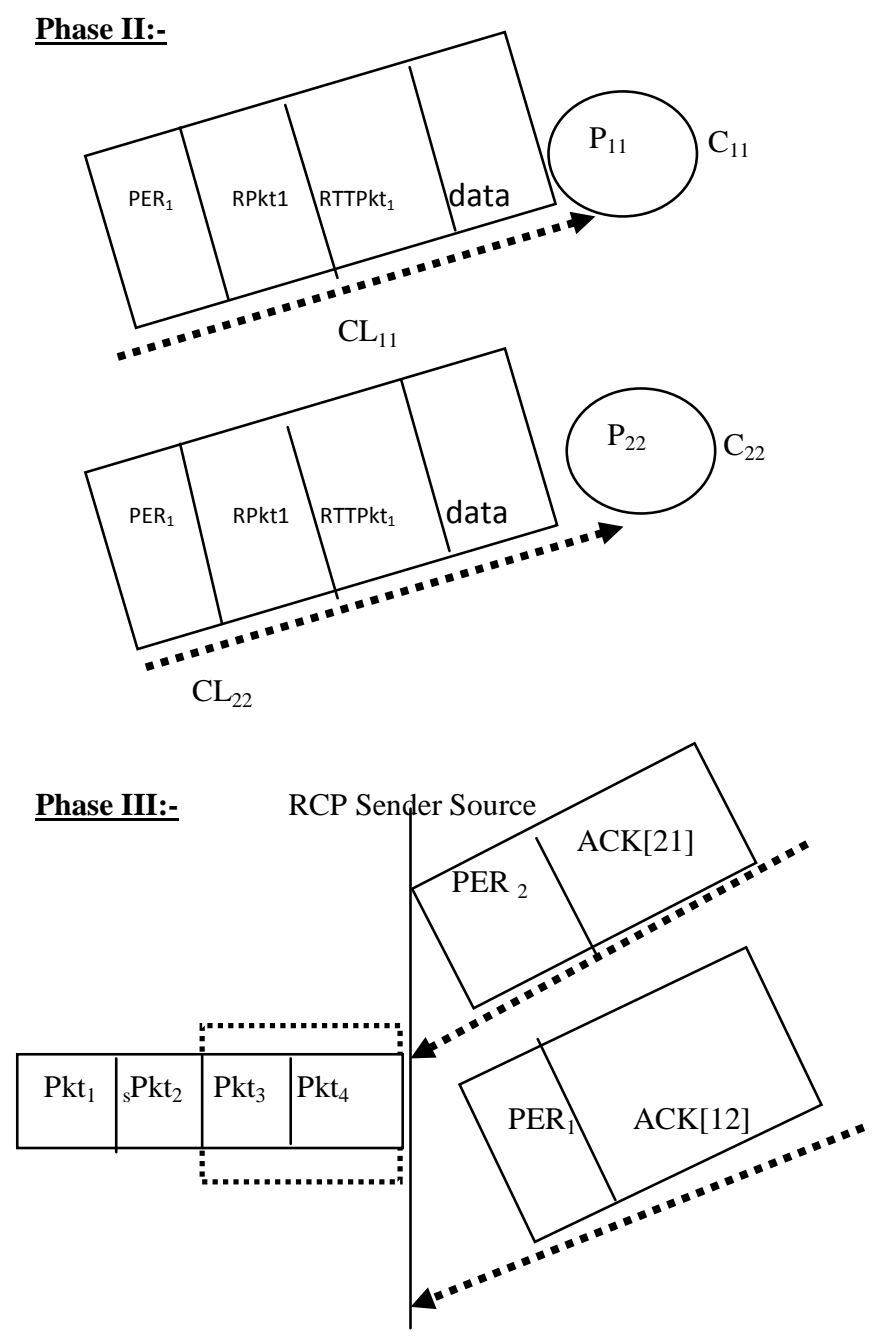

(a),(b)\&(c)

Fig 2: Algorithm Phases Illustration

\section{CONCLUSIONS}

In this paper we have proposed a model and mechanism for congestion control using window size, Queue length and link capacity with accelrating effect over capacity based wireless network. We also tries to identify the cases for reasons of congestion with different values of link capacity, processing capacity of nodes with over all effect of accelration mechanism and tries to provide solution for them by proposed algorithm with illustration of three phases and their steps.

\subsection{Future Scope}

In this paper the proposed model and mechanism for congestion control is efficient and effective over wireless network but the proposed model further may be improved by more effective queuing technique, window management technique and accelration mechanism over wireless network and ad-hoc network. This proposed model further extended with reference to scalability feature.

\section{REFERENCES}

[1] Dukkipati, N., Kobayashi, M., Zhang-Shen, R., and Nick McKeown, N. 2005 Processor Sharing flows in the Internet", In Thirteenth International Workshop on Quality of Service (IWQoS).

[2] Munir, A., Member, Qaisar, S. and, Member 2010 Coded Rate Control Protocol (C-RCP) for Lossy Channels. In $44^{\text {th }}$ IEEE Annual Conference on Information Sciences and Systems (CISS 2010).

[3] Jain, S., Loguinov, D. 2007 PIQI-RCP: Design and Analysis of Explicit Congestion Control . In $15^{\text {th }}$ International Work shop on Quality of Service (IEEE IWQoS 2007) .

[4] Dukkipati, N., McKeown, N., and, Fraser, A. G. 2006 RCP-AC: Congestion control to make flows complete quickly in any Environment. In Proceedings of the INFOCOM $25^{\text {th }}$ IEEE International Conference Computer Communications.

[5] Sridharan, A., and Krishnamachari, B. 2009 Explicit and Precise Rate Control for Wireless Sensor Networks Networks.

[6] en.wikipedia.org/wiki/Wireless_network.

[7] Kelly, F., Raina, G., and, V. Thomas. 2008 Stability and Fairness of Explicit Congestion Control with Small Buffers.

[8] Balakrishnan, H., Dukkipati, N., McKeown, N. and, Tomlin, C. J. 2007 Stability analysis of explicit congestion control protocols", In Proceedings IEEE Commun. Letter, vol. 11,

[9] Katabi, D., Handley, D. M. and C. Rohrs 2002 Congestion control for high bandwidth-delay product networks",Computer Communication.

[10] Rangwala, S., Jindal, A., Jang, K.Y., Psounis, K. and R.Govindan 2008.Understanding congestion control in Multihop wireless mesh networks.

[11] Varshney,U. and, Jain ,R. 2001 .Issues in Emerging 4G Wireless Networks", In proceedings of the IEEE Comm. Letter.

[12] Dukkipati, N. and, McKeown ,N. 2006 Why flow completion time is the right metric for congestion control In ACM SIGCOMM Computer Communication Review.

[13] S.Hauger, M. Scharf, J. Kogel, C. Suriyajan, "Evaluation of Router Implementations for Explicit Congestion Control Schemes ", Journal of Communications ,2010, 197-204.

[14] Luby, M. 2002 LT codes .In Proceedings of IEEE Symposium on Foundations of Computer Science (FOCS).

[15] Dr. E.Chandra, and B.Subramani, "A Survey on CongestionControl", Global Journal of Computer Science and Technology ", 2010, 82-87

[16] Akyildiz, I.F., and Wang, X. 2005 A survey on wireless mesh Networks .In Proceedings of IEEE Radio Communications.

[17] “100x100 clean state project." [Online]. Available:http:// 100x100network.org/ 


\section{AUTHORS PROFILE}

Vivek Singhal is working as Assistant Professor at College of Engineering Roorkee, Roorkee (U.K.).He is MTech. (C.E.) from Shobhit University Meerut and B-Tech (C.S.) from College of Engineering Roorkee, Roorkee. He has around 6 years teaching experience. He has been member of several academic and administrative bodies. He has attended several seminars, workshops and conferences. His paper was published in National conference across India. His area of research includes Wireless Networks, Congestion Control, and Artificial Intelligence.

A.K.Vatsa is working as Assistant Professor in the School of Computer Engineering and Information Technolo-gy at Shobhit University, Meerut (U.P)He obtained his M-Tech(C.E.) from Shobhit University and B-Tech(I.T.) from V.B.S. Purvanchal
University, Jaunpur(U.P.). He has worked as software engineer in software industry. He has been in teaching for the past one decade. He has been member of several academic and administrative bodies. During his teaching he has coordinated several Technical fests and National Conferences at Institute and University Level. He has attended several seminars, workshops and conferences at various levels. His several papers are in various national and international conferences across India. His area of research includes MANET (Mobile Ad-Hoc network), Congestion Control, Network Security and VOIP-SIP (Voice over IP) 